\title{
Les médecins en faveur de l'environnement
}

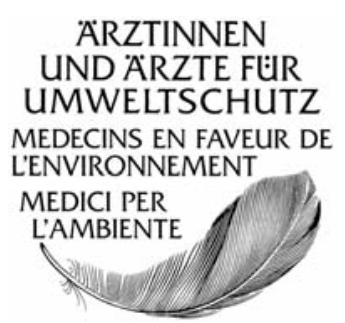

Depuis 21 ans déjà - date de la fondation de leur association - les médecins en faveur de l'environnement (MfE) sensibilisent le corps médical, les patients et la population aux risques de santé liés à l'environnement et s'engagent également en politique. Ils se considèrent comme les représentants du domaine spécifique de la médecine environnementale et, en même temps, comme une organisation non gouvernementale indépendante.

\section{Dr Peter Kälin,}

président

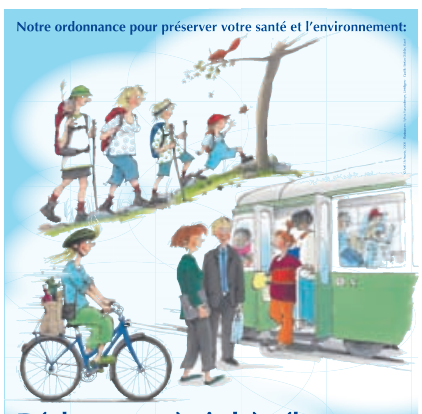

Déplacez-vous à pied, à vélo et en transports publics afin de freiner le réchauffement climatique et d'améliorer la qualité de l'air que nous respirons.

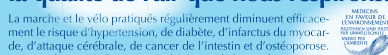

Correspondance:

Médecins en faveur

de l'environnement (MfE)

Case postale 111

CH-4013 Bâle

Tél. 0613224949

info@aefu.ch

www.aefu.ch

\section{La pollution de I'air reste un sujet important}

L'association est née de la préoccupation d'une pollution de l'air toujours plus importante et de ses effets sur la santé. Au cours des dernières décennies, ce sujet a fortement marqué les activités de l'association dont la brochure «Pollution de l'air et santé», scientifiquement fondée et facilement compréhensible, est le document de base utilisé dans ce domaine. Sa nouvelle documentation sur les particules fines et le smog estival formule des exigences envers les autorités et les politiques et fournit des conseils à la population pour que chacun contribue à améliorer la qualité de l'air. La nouvelle affiche pour salle d'attente, visant à promouvoir l'utilisation des transports publics ou du vélo ainsi que la marche à pied en vue de freiner le réchauffement climatique et d'améliorer la qualité de l'air, résume les principales recommandations des MfE.

L'obligation d'introduire des filtres à particules pour les moteurs diesel et le transfert du transport routier au rail fait également partie de nos revendications.

\section{Projet pilote: nouveau réseau de conseil en médecine environnementale}

Les questions posées par les personnes cherchant de l'aide le prouvent: le besoin de conseil en médecine environnementale, comprenant un éventuel examen médical et, si nécessaire, une analyse approfondie (par ex. investigations à l'intérieur de l'habitat) est bien réel. Les MfE ont saisi la balle au bond et mis sur pied un nouveau projet pilote. Une évaluation indépendante complète et compétente est proposée aux patients qui supposent que des facteurs environnementaux sont à l'origine de leurs maux. Le réseau de conseil en médecine environnementale se compose d'un centre de coordination et de médecins de premiers re- cours qui proposent des examens médicaux (standardisés selon un protocole uniforme) tenant compte à égalité des aspects somatiques, psychiques et environnementaux. En cas de besoin, ils font appel à des spécialistes médicaux ou environnementaux. L'institut de médecine sociale et préventive (ISPM) de l'Université de Berne se charge du suivi scientifique du projet.

\section{Activités diversifiées au sein du comité central, des sections et des groupes de travail}

L'individu, à lui seul, ne peut guère échapper aux effets négatifs liés à l'environnement. C'est pourquoi, il est nécessaire que la société agisse. Les médecins sont en mesure de reconnaître les effets de la pollution et d'agir dans l'intérêt de la population. Les quelque 1800 membres que compte notre association lui fournissent le poids nécessaire pour y parvenir. Au sein de nos divers comités, des médecins engagés traitent de sujets aussi différents que les changements climatiques, l'air, les nuisances sonores, les questions énergétiques, les champs électromagnétiques, le génie génétique et la manière de gérer un cabinet médical dans le respect de l'environnement.

Outre l'engagement politique, notre association accorde une importance particulière à la formation continue de ses membres. Notre session annuelle de formation continue intitulée «Médecine et environnement» offre un approfondissement des connaissances pratiques. La revue Ecoscope - qui paraît une fois par année en français et tous les trimestres en allemand - propose des articles spécialisés traitant de questions sanitaires et environnementales. Les MfE font le lien entre connaissances scientifiques et pratiques et en informent leurs membres. Nous souhaitons la bienvenue à toute nouvelle personne qui désire s'affilier. 